ICRR-Report-675-2014-1

IPMU14-0088

\title{
Isocurvature perturbations and tensor mode in light of Planck and BICEP2
}

\author{
Masahiro Kawasaki ${ }^{1,2}$, Toyokazu Sekiguchi ${ }^{3}$, Tomo Takahashi ${ }^{4}$ \\ and Shuichiro Yokoyama ${ }^{1}$ \\ 1 Institute for Cosmic Ray Research, University of Tokyo, Kashiwa 277-8582, Japan \\ ${ }^{2}$ Kavli Institute for the Physics and Mathematics of the Universe (Kavli IPMU), \\ TODIAS, the University of Tokyo, 5-1-5 Kashiwanoha, Kashiwa, 277-8583, Japan \\ ${ }^{3}$ Helsinki Institute of Physics, University of Helsinki, PO Box 64, FIN-00014, Finland \\ 4 Department of Physics, Saga University, Saga 840-8502, Japan
}

\begin{abstract}
We investigate the degeneracy of the isocurvature perturbations and the primordial gravitational waves, by using recent observations of the cosmic microwave background (CMB) reported by Planck and BICEP2 collaborations. We show that the tension in the bound on the tensor-to-scalar ratio $r$ between Planck and BICEP2 can be resolved by introducing the anti-correlated isocurvature perturbations. Quantitatively, we find that with the anti-correlated isocurvature perturbations the constraints on $r$ from Planck alone and BICEP2 results can be consistent at $68 \%$ C.L.
\end{abstract}




\section{Introduction}

Recently the detection of primordial gravitational waves has been reported by BICEP2 [1, 2]. The size of the gravitational waves is usually characterized by the so-called tensor-toscalar ratio $r$ and its reported value from BICEP2 is

$$
r=0.2_{-0.05}^{+0.07} \quad(68 \% \text { C.L. }) \text {, }
$$

which excludes $r=0$ with $7 \sigma$ [1]. However, this large value of $r$ is in strong tension with the temperature data from Planck in the framework of the standard $\Lambda \mathrm{CDM}+r$ model which gives the limit $r<0.11$ [3]. Some possible extensions of the model to resolve the tension have been discussed since the data release of BICEP2 such as the running spectral index [1, 4, 5], the suppression of the adiabatic perturbations on large scales [6] 9], the presence of the extra relativistic particles [10,11, blue-tilted tensor spectrum [12 14, the existence of anti-correlated isocurvature mode [15,16], and so on. Among these possibilities, we in this paper focus on the model with isocurvature perturbations. Since the constraints on $r$ from the temperature data come from the enhancement due to the tensor mode contribution on large scales, if one can reduce the power on large scales, a large tensor mode contribution can be accommodated, which can be realized by assuming the anti-correlated isocurvature mode since it suppresses the TT power spectrum on large scales. Since such anti-correlated isocurvature perturbations can naturally arise in multiple scalar field models such as the curvaton $17-19 \#$, this issue deserves a further study. In this paper, we investigate how the tension can be reduced in a more quantitative way by performing Markov Chain Monte Carlo (MCMC) analysis using Planck, BICEP2 and some other data. To discuss the effects of a broad class of models with isocurvature perturbations, we study not only a case with anti-correlated isocurvature mode, but also a generally correlated one, as well as varying the isocurvature spectral index. As will be discussed, the existence of the anticorrelated isocurvature mode can allow large contribution from the tensor mode consistent with Planck temperature data, which may give a hint of the existence of the isocurvature mode.

It should also be noted that constraints on isocurvature perturbations in the light of BICEP2 data are worth investigating on their own. Although the constraints on isocurvature perturbations have been studied in many works, to our knowledge, there are only a few studies on isocurvature perturbations in the existence of the tensor mode [25 27]. Now the BICEP2 data indicates that the tensor mode should not be neglected, the investigation of isocurvature perturbations in the presence of the tensor mode would be an important subject.

This paper is organized as follows. In the next section, we briefly give a formalism to discuss the isocurvature perturbations and the effects of isocurvature perturbations and tensor mode in the CMB power spectra. Then in Section 3, we describe the method of the analysis. In Section 4, we show our results and discuss how the tension between Planck

\footnotetext{
\#1 Isocurvature perturbations in the curvaton model have been discussed in [20, 24].
} 
and BICEP2 data can be resolved by introducing isocurvature perturbations. The final section is devoted to the conclusion of this paper.

\section{Effects of isocurvature perturbations and the ten- sor mode}

\subsection{Definitions}

First we summarize the formalism to discuss isocurvature perturbations and set our notations. Isocurvature perturbations for a component $i$ is usually defined as

$$
S_{i} \equiv 3\left(\zeta_{i}-\zeta_{r}\right)
$$

where $\zeta_{i}$ is the curvature perturbation on the uniform energy density hypersurface of $i$ and the subscript $r$ indicates radiation. Although various isocurvature modes have been discussed in the literature (see Ref. 3 and references therein), we only consider cold dark matter (CDM) mode in this paper \#2. Thus in the following, we denote $S_{\mathrm{CDM}}$ simply as $S$ and also the adiabatic curvature perturbations during radiation dominated era $\zeta_{r}$ simply as $\zeta$. Depending on the model, the curvature and isocurvature perturbations can be correlated or uncorrelated.

The power spectrum of the initial perturbations $P_{X Y}(k)$ is defined by $\left\langle X(\vec{k}) Y\left(\vec{k}^{\prime}\right)\right\rangle=$ $P_{X Y}(k)(2 \pi)^{3} \delta^{(3)}\left(\vec{k}+\vec{k}^{\prime}\right)$ with $X$ and $Y$ being either $\zeta$ or $S$. To discuss the size and the scaledependence of the curvature (adiabatic) and isocurvature perturbations, we characterize the power spectra for the auto- and cross-correlation of $\zeta$ and $S$ as

$$
\begin{gathered}
\mathcal{P}_{\zeta}(k)=\frac{k^{3}}{2 \pi^{2}} P_{\zeta \zeta}(k)=A_{\zeta}\left(k_{0}\right)\left(\frac{k}{k_{0}}\right)^{n_{s}-1}, \\
\mathcal{P}_{S}(k)=\frac{k^{3}}{2 \pi^{2}} P_{S S}(k)=A_{S}\left(k_{0}\right)\left(\frac{k}{k_{0}}\right)^{n_{\mathrm{iso}}-1}, \\
\mathcal{P}_{\zeta S}(k)=\frac{k^{3}}{2 \pi^{2}} P_{\zeta S}(k)=A_{\zeta S}\left(k_{0}\right)\left(\frac{k}{k_{0}}\right)^{n_{c}-1},
\end{gathered}
$$

where $A_{\zeta}, A_{S}$ and $A_{\zeta S}$ are respectively the amplitude of the power spectra, $n_{s}, n_{\text {iso }}$ and $n_{c}$ are spectral indices for the auto-correlation power spectra of $\zeta, S$ and the cross-correlation one, respectively. We define these quantities at the reference scale $k_{0}$ which we adopt $k_{0}=0.05 \mathrm{Mpc}^{-1}$ for the analysis in this paper.

Depending on the model, the degree of the correlation between the adiabatic and isocurvature perturbations varies. To quantify the cross-correlation, we define the correlation

\#2 Since the effects of the baryon isocurvature mode on the CMB power spectrum are identical with the CDM one, our results can also be translated into the baryonic mode by rescaling its amplitude in proportion to their energy densities. 
parameter as

$$
\gamma_{\text {iso }} \equiv \frac{P_{\zeta S}\left(k_{0}\right)}{\sqrt{P_{\zeta}\left(k_{0}\right) P_{S}\left(k_{0}\right)}} .
$$

We denote the cases with $\gamma_{\text {iso }}>0, \gamma_{\text {iso }}<0$ and $\gamma_{\text {iso }}=0$ as positively correlated, negatively (anti-) correlated and uncorrelated isocurvature perturbations, respectively. Although the spectral indices can be generally treated as independent parameters, in this paper, we assume the case with $n_{s}=n_{\text {iso }}=n_{c}$, which is motivated by the curvaton model where the adiabatic and isocurvature perturbations originate from a single scalar field. When we consider the case with uncorrelated isocurvature mode, we also investigate a possibility of $n_{s} \neq n_{\text {iso }}$ which is the case for the axion isocurvature mode $\# 3$.

In the analysis, to parameterize the size of isocurvature perturbations, the fraction of their contribution is commonly used and it is defined as

$$
\alpha_{\text {iso }} \equiv \frac{P_{S}\left(k_{0}\right)}{P_{\zeta}\left(k_{0}\right)+P_{S}\left(k_{0}\right)}
$$

Since we consider cases with isocurvature perturbations, we define the tensor-to-scalar ratio including the isocurvature one in the scalar part as

$$
r \equiv \frac{P_{T}\left(k_{0}\right)}{P_{\zeta}\left(k_{0}\right)+P_{S}\left(k_{0}\right)},
$$

where $P_{T}$ denotes the power spectrum of the tensor mode. Besides, we assume a scaleinvariant tensor power spectrum, i.e. $P_{T}(k) k^{3}=$ const, throughout this paper.

\subsection{Effects on CMB}

The contribution of the adiabatic and isocurvature modes to temperature fluctuations can be roughly evaluated by looking at those for the Sachs-Wolfe (SW) effect on large scales (see, e.g., Ref. [29]):

$$
\left.\frac{\Delta T}{T}\right|_{\mathrm{SW}}=-\frac{1}{5} \zeta-\frac{2}{5} S .
$$

Thus the CMB TT angular power spectrum from the SW effect can be written as

$$
\left.\frac{l(l+1) C_{l}^{\mathrm{TT}}}{2 \pi}\right|_{\mathrm{SW}} \propto \mathcal{P}_{\zeta}+4 \mathcal{P}_{\zeta S}+4 \mathcal{P}_{S} .
$$

When the correlation parameter is $\gamma_{\text {iso }}<0, \mathcal{P}_{\zeta S}$ can give a negative contribution to the SW effect, which suppresses the power on large scales. In Fig. 1, CMB TT power spectra are shown for the cases with adiabatic, anti-correlated isocurvature and tensor modes. As shown in the figure, the spectrum for the anti-correlated isocurvature case is suppressed on large scale, which can cancel the tensor mode contribution. Thus we expect that

\#3 While in many models $n_{\text {iso }}$ is close to 1 , in some models $n_{\text {iso }}$ can be as large as $n_{\text {iso }} \simeq 4[28$. 
the inclusion of the anti-correlated isocurvature mode would help to resolve the tension between the temperature data from Planck and the observation of B-mode polarization from BICEP2.

In the next section, we study this issue in a more quantitative way by using a MCMC analysis to fit the model with isocurvature perturbations and tensor mode.

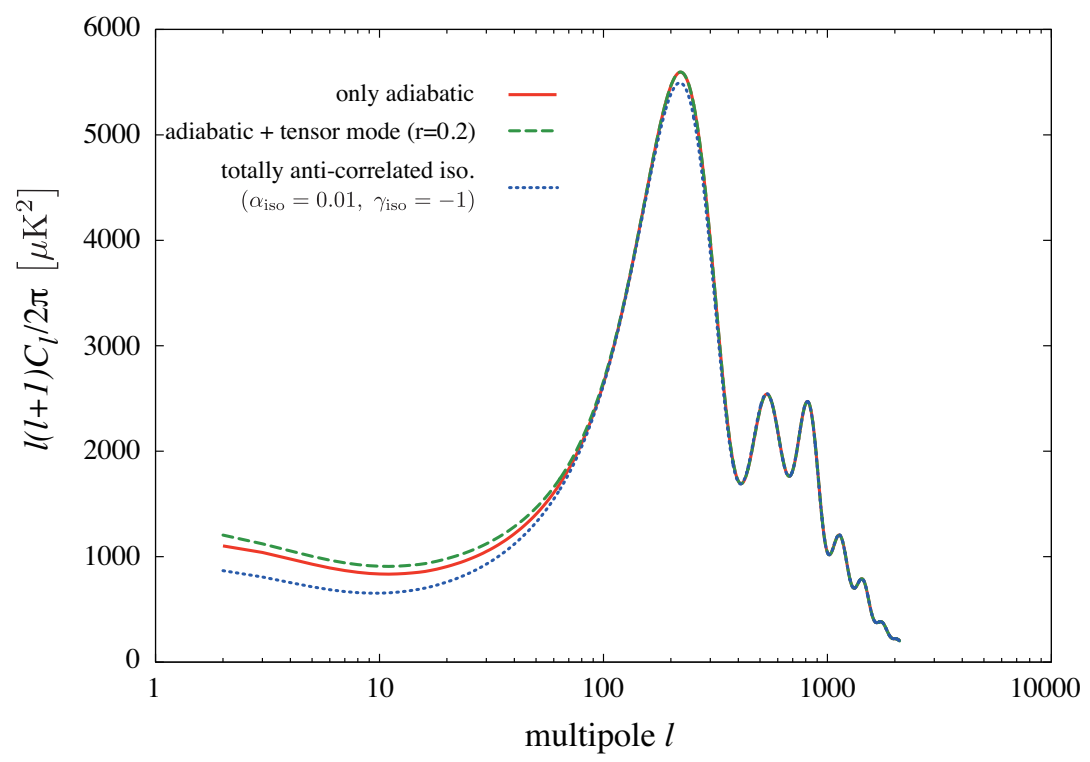

Figure 1: CMB TT power spectrum for the cases with only adiabatic (red solid line), adiabatic and totally anti-correlated isocurvature $\left(\alpha_{\text {iso }}=0.01, \gamma_{\text {iso }}=-1\right)$ (blue dotted line), and adiabatic and tensor modes with $r=0.2$ (green dashed line). For other cosmological parameters, here we adopt the best-fit values obtained for a flat $\Lambda$ CDM model by Planck and WMAP 9-year polarization data [30].

\section{Analysis}

In our analysis, we assume a flat $\Lambda$ CDM model with isocurvature perturbations and a tensor mode, which consists of the following cosmological parameters

$$
\left(\Omega_{b} h^{2}, \Omega_{c} h^{2}, H_{0}, \tau, n_{s}, A_{\zeta}, r, \alpha_{\text {iso }}, \gamma_{\text {iso }}, n_{\text {iso }}, n_{c}\right),
$$

where $\Omega_{b} h^{2}$ and $\Omega_{c} h^{2}$ respectively are the density parameters of baryon and CDM, $H_{0}=$ $100 \mathrm{~h}$ is the Hubble parameter in units of $\mathrm{km} / \mathrm{sec} / \mathrm{Mpc}, \tau$ is the optical depth of reionization. However, we do not vary all of these parameters completely independently. Instead, we often treat some of the parameters for the initial power spectrum as fixed ones or related in some way (e.g., we assume $n_{s}=n_{\text {iso }}=n_{c}$ for some analysis). 
We adopt the latest version of CosmoMC [31,32], which allows us to sample the posterior distribution of the cosmological parameters as well as various nuisance parameters which account for the contributions of foregrounds to CMB.

As CMB data, we adopt the temperature anisotropy from Planck (hereafter P13) [30, 33, 34, the E-mode polarization one from WMAP 9-year (WP9) [35, 36] and the E- and B-mode polarization ones from BICEP2 (B2) [1, 2]\#4. In some cases we also combine the measurements of the baryon acoustic oscillation scales [37 39] (BAO) and the direct measurement of the Hubble parameter [40] (H0).

\section{Result}

Now we present our result. In Table 1, we summarize constraints on cosmological parameters related to the tensor and isocurvature perturbations for various models, which we are to describe in the following.

Let us first see the constraints on isocurvature models with definite correlations $\gamma_{\text {iso }}=0$, 1 and -1 , which we refer to as uncorrelated, totally correlated and totally anti-correlated cases, respectively. Here we assumed $n_{\text {iso }}=n_{c}=n_{s}$. In Fig. 2, we show constraints in the $r$ - $\alpha_{\text {iso }}$ plane obtained from three combinations of data sets P13+WP9, P13+WP9+B2, and $\mathrm{P} 13+\mathrm{WP} 9+\mathrm{B} 2+\mathrm{BAO}+\mathrm{H} 0$. This figure enables us to see what types of the correlation of the isocurvature perturbations parameterized by $\gamma_{\text {iso }}$ can weaken the discrepancy in the constraints on $r$ between the Planck and BICEP2 data. In the totally anti-correlated case, there arise an overlap between the allowed regions of P13+WP9 and P13+WP9+B2 at 68 $\%$ C.L., and a large overlap at 95\% C.L. level. Besides, we also computed the minimum values of $\chi^{2}$ in both the purely adiabatic and anti-correlated isocurvature models. We found that inclusion of anti-correlated isocurvature perturbations can significantly improve the fit to data; the minimum $\chi^{2}$ decreases by 6.6 (5.8) for the data set P13+WP2+B2 $(\mathrm{P} 13+\mathrm{WP} 2+\mathrm{B} 2+\mathrm{BAO}+\mathrm{H} 0)$. These results indicate that, in the presence of totally anticorrelated isocurvature perturbations, the tension between the Planck and BICEP2 data can be reduced. In Fig. 3, we compare the best-fit CMB power spectra of the purely adiabatic and anti-correlated cases to the data P13+WMAP9+B2+BAO+H0. We note that in these two best-fit models $\left(\alpha_{\text {iso }}, r\right)$ takes the values $(0,0.17)$ and $(0.0036,0.18)$. We can see the anti-correlated isocurvature model can give a lower TT power at large angular scales and a better fit to the Planck data than the purely adiabatic model.

On the other hand, in the case of uncorrelated or totally (positively) correlated isocurvature mode, allowed regions at $68 \%$ C.L. for data with and without BICEP2 do not overlap with each other (see Fig. 2). We also found that in these cases, the best-fit points to data are obtained near $\alpha_{\text {iso }}=0$ and there are no significant reductions of the minimum $\chi^{2}$ from the purely adiabatic case for all the data sets we adopted.

${ }^{\# 4}$ Note that we combine the polarization data of WP9 and B2 by simply adding $\chi^{2}$ from the likelihood codes of them. This is justified since these two data are dominated by the measurement noise and the sample variance is subdominant. 


\begin{tabular}{|c|c|c|c|c|}
\hline model & data & \multicolumn{3}{|c|}{ parameter } \\
\hline purely adiabatic & $\begin{array}{l}\mathrm{P} 13+\mathrm{WP} 9 \\
\quad+\mathrm{B} 2 \\
\quad+\mathrm{B} 2+\mathrm{BAO}+\mathrm{H} 0\end{array}$ & 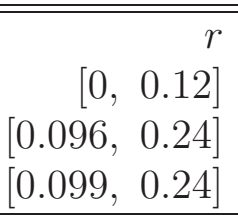 & & \\
\hline uncorrelated & $\begin{array}{l}\mathrm{P} 13+\mathrm{WP} 9 \\
\quad+\mathrm{B} 2 \\
\quad+\mathrm{B} 2+\mathrm{BAO}+\mathrm{H} 0\end{array}$ & 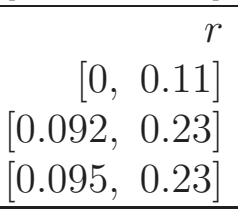 & 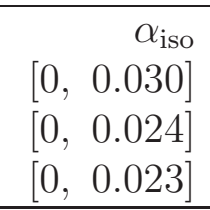 & \\
\hline totally correlated & $\begin{array}{l}\mathrm{P} 13+\mathrm{WP} 9 \\
\quad+\mathrm{B} 2 \\
\quad+\mathrm{B} 2+\mathrm{BAO}+\mathrm{H} 0\end{array}$ & 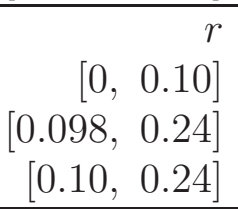 & $\begin{array}{lr} & \alpha_{\text {iso }} \\
{[0,} & 0.0026] \\
{[0,} & 0.0015] \\
{[0,} & 0.0015]\end{array}$ & \\
\hline totally anti-correlated & $\begin{array}{l}\mathrm{P} 13+\mathrm{WP} 9 \\
\quad+\mathrm{B} 2 \\
\quad+\mathrm{B} 2+\mathrm{BAO}+\mathrm{H} 0\end{array}$ & 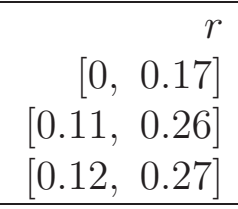 & 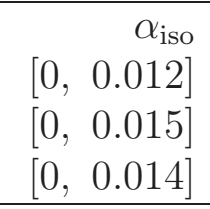 & \\
\hline generally correlated & $\begin{array}{c}\mathrm{P} 13+\mathrm{WP} 9+\mathrm{B} 2 \\
+\mathrm{BAO}+\mathrm{H} 0\end{array}$ & $\begin{array}{r}r \\
{[0.10,0.25]} \\
{[0.11,0.26]}\end{array}$ & 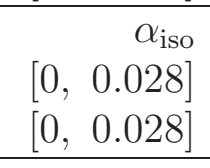 & 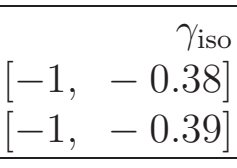 \\
\hline varying $n_{\text {iso }}$ (uncorrelated) & $\begin{array}{c}\mathrm{P} 13+\mathrm{WP} 9+\mathrm{B} 2 \\
+\mathrm{BAO}+\mathrm{H} 0 \\
\end{array}$ & 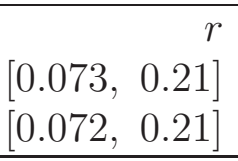 & 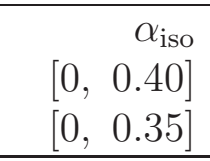 & $\begin{array}{r}n_{\text {iso }} \\
{[1.9,4]} \\
{[1.9,4]}\end{array}$ \\
\hline
\end{tabular}

Table 1: Constraints on cosmological parameters related to the tensor and isocurvature perturbations. Shown are the $95 \%$ intervals of 1D marginalized posterior distributions for various model and combinations of data sets. 

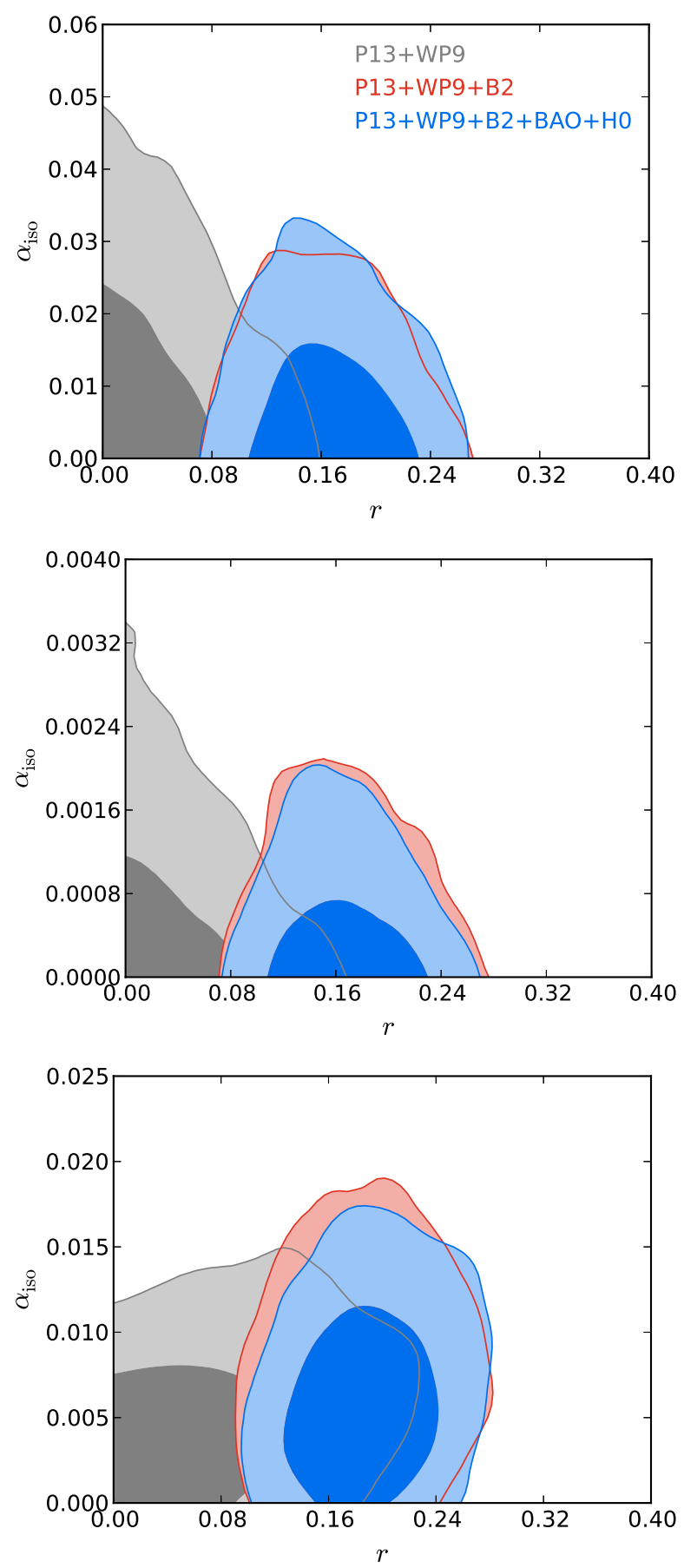

Figure 2: 2D Constraints (68\% and 95\% C.L.) in the $r$ - $\alpha_{\text {iso }}$ plane for the cases of fixed $\gamma_{\text {iso }}=0$ (top), 1 (middle) and -1 (bottom). Note that scales in $y$-axis differ significantly. 

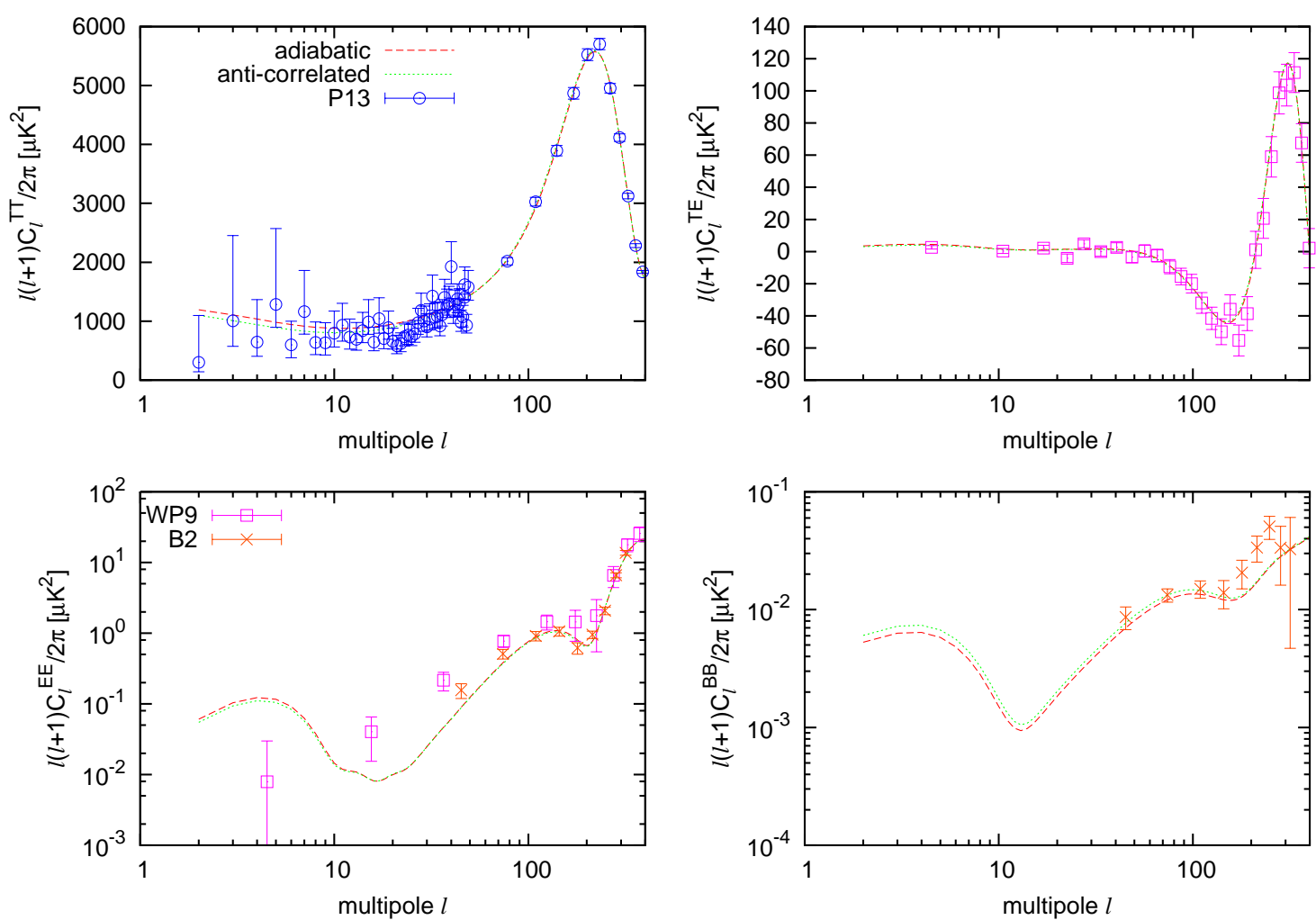

Figure 3: Best-fit lensed CMB power spectra of the adiabatic (dashed red) and anticorrelated isocurvature (dotted green) models to the data $\mathrm{P} 13+\mathrm{WP} 9+\mathrm{B} 2+\mathrm{BAO}+\mathrm{H} 0$. The TT (top left), TE (top right), EE (bottom left) and BB (bottom right) power spectra are shown. Data points depicted are P13 (TT), WP9 (TE, EE) and B2 (EE, BB). Note that the $y$-axises in the top and bottom panels are shown in linear and logarithmic scales, respectively. 

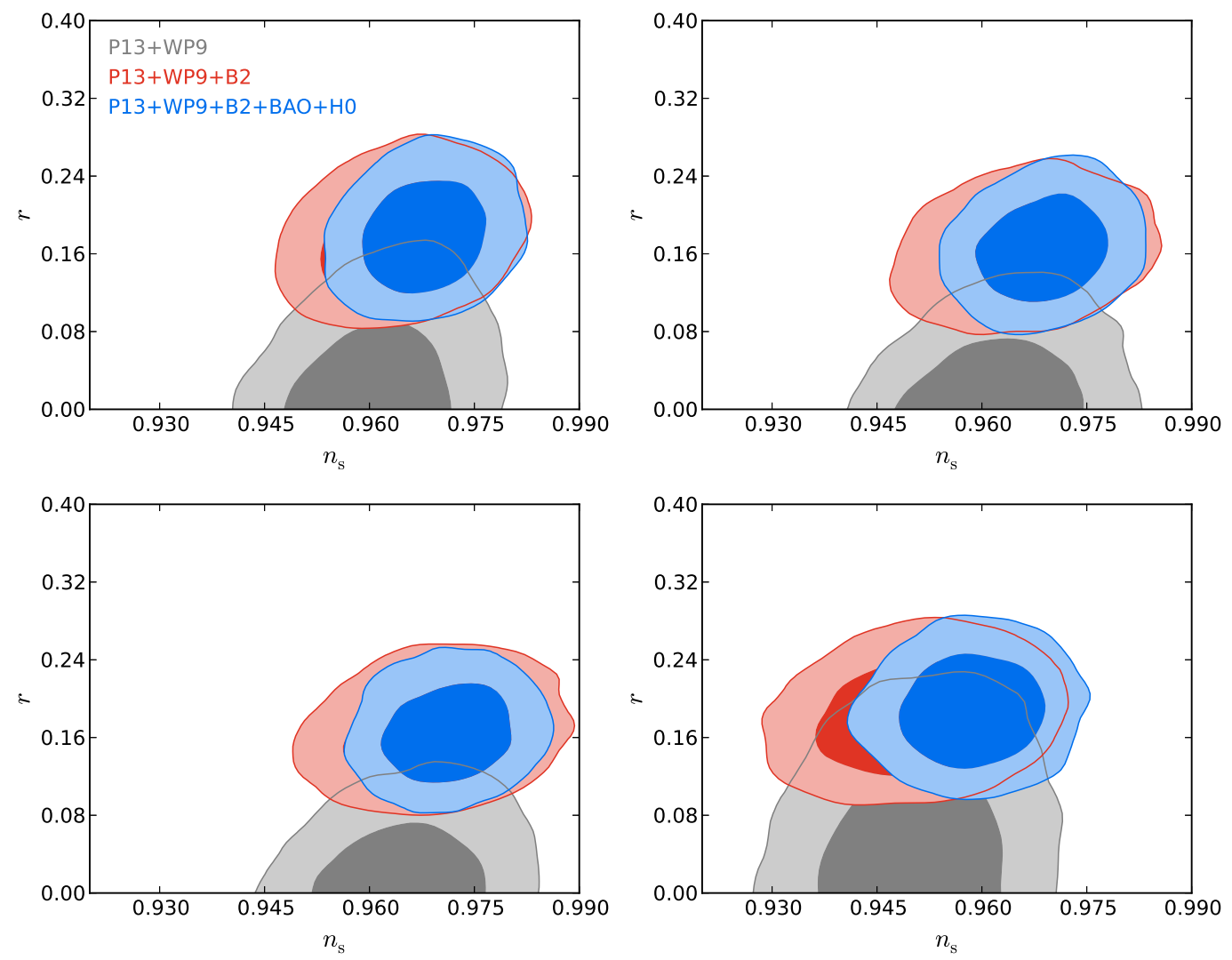

Figure 4: 2D Constraints in the $n_{s}-r$ plane for the cases without isocurvature perturbations (top left), with isocurvature ones for $\gamma_{\text {iso }}=0$ (top right), 1 (bottom left) and -1 (bottom right). 
In Fig. (4, we also show the constraints on the $n_{s}-r$ plane for the cases with no isocurvature (only adiabatic) perturbations, totally (positively) correlated, anti-correlated and uncorrelated isocurvature ones. The existence of anti-correlated isocurvature perturbations makes the Planck data to be consistent with larger tensor mode indicated by BICEP2. This figure also clearly shows that the tension between Planck and BICEP2 can be weakened by introducing the anti-correlated isocurvature fluctuations.

Next, we consider a case of generally correlated isocurvature perturbations (hereafter we denote as "generally correlated case"), where $\gamma_{\text {iso }}$ can vary in the range $[-1,1]$. Here we again assume $n_{\text {iso }}=n_{c}=n_{s}$. In Fig. 5, we show constraints in the $r$ - $\alpha_{\text {iso }}$ and the $\gamma_{\text {iso }}-\alpha_{\text {iso }}$ planes. From the figure, one clearly sees there is a strong preference for $\gamma_{\text {iso }}=-1$, which is exactly what we expect from the previous result. In addition, we found that the best-fit points of the generally correlated case are located near $\gamma_{\text {iso }}=-1$ and there are no significant changes in the minimum value of $\chi^{2}$ from the totally anti-correlated case. However, this does not mean $\gamma_{\text {iso }}$ can not differ significantly from -1 and actually $\gamma_{\text {iso }} \simeq-0.4$ is still allowed, though in such a case the improvement in the fit from the purely adiabatic case should be somewhat less prominent. This is of particular importance when one considers a model with anti-correlated isocurvature and tensor perturbations. In fact, to generate isocurvature perturbations, the existence of a light scalar field other than the inflaton is required. In such a case, isocurvature perturbations of the light scalar field can not correlate with the inflaton ones. Thus, to obtain largely correlated isocurvature perturbations, the contribution from the other scalar field to the adiabatic perturbations should be large. Furthermore, when the correlation between adiabatic and isocurvature ones is relatively large, the tensor-to-scalar ratio tends to be suppressed than in the case where the adiabatic perturbations are sourced only from the inflaton, $r=r_{\text {inf }}\left(1-\alpha_{\text {iso }}\right)\left(1-\gamma_{\text {iso }}^{2}\right)\left(r_{\text {inf }}\right.$ : the tensor-to-scalar ratio for inflaton only). Hence, in order to realize $\left|\gamma_{\text {iso }}\right| \simeq 1$, we need to consider the situation where the inflaton contribution to the adiabatic curvature perturbations is relatively small and the tensor-to-scalar ratio is reduced. We are able to construct a theoretical model based on e.g. the curvaton scenario, which allows to generate enough anti-correlated isocurvature and tensor perturbations as discussed in Ref. [15].

So far we have seen that the presence of anti-correlated isocurvature perturbations can greatly reduce the tension between the Planck and BICEP2 data. Then one may wonder if this means that nonzero isocurvature perturbations are suggested by the combination of Planck and BICEP2. However, this conclusion can not be drawn from the current data. As is given in Table 1 and also seen from Figs. 2 and 5, the current data is still consistent with $\alpha_{\text {iso }}=0$ at $95 \%$ C.L.

Then how can we confirm the anti-correlated isocurvature model? To discuss this issue, we look for combinations of cosmological parameters which significantly degenerate with $\alpha_{\text {iso }}$ in the anti-correlated cases. We found that $A_{\zeta} e^{-2 \tau}$, which determines the amplitudes of the CMB power spectra (except for the tensor contribution), is one of the most degenerate quantities to $\alpha_{\mathrm{iso}}$, as shown in Fig. 6. We expect precise measurements of the TE and EE power spectra can improve the determination of $A_{\zeta} e^{-2 \tau}$ and may pin down nonzero $\alpha_{\text {iso. }}$. In 
the near future, this will be achieved by the Planck polarization measurement [42], which will also determine $\tau$ alone better and hence may help to break the parameter degeneracy further. In addition, since the anti-correlated isocurvature model prefers larger $r$ compared to the purely adiabatic model, precise measurements of the BB power spectrum would also strengthen (or weaken) the need for anti-correlated isocurvature perturbations. Planck can measure $r$ with an accuracy $\Delta r \simeq$ a few percents [42. The precision can be improved further by proposed CMB surveys such as Lite-Bird [43, PIXIE [44 and PRISM [45], which will focus on the B-mode polarization at large angular scales.
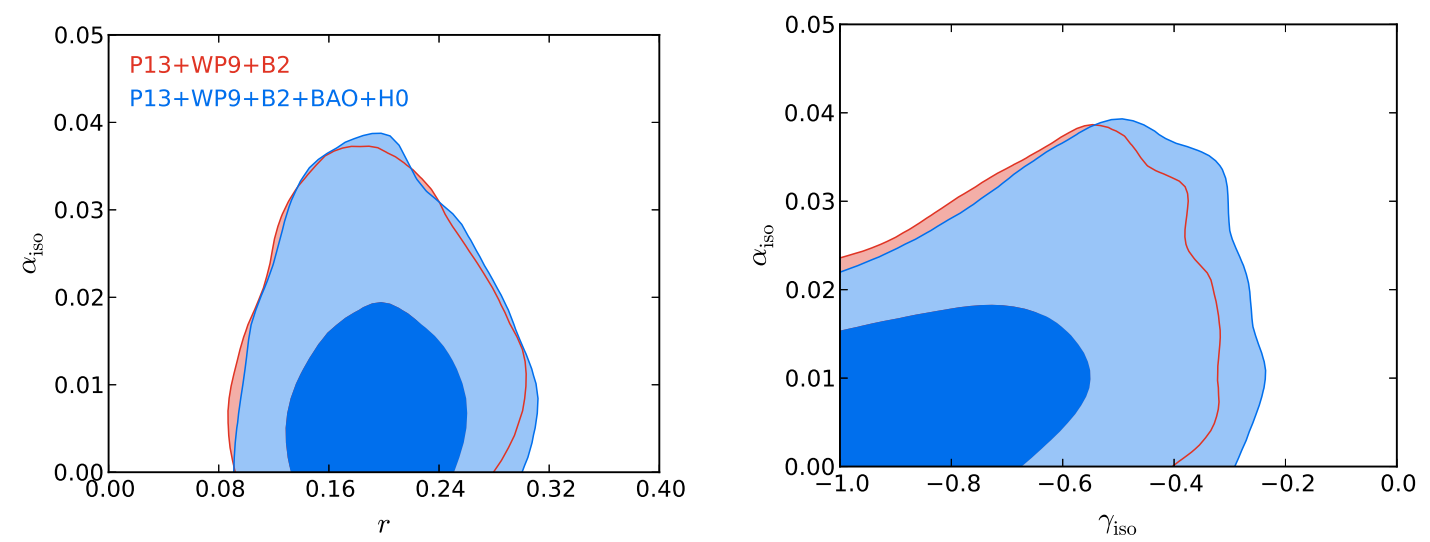

Figure 5: 2D constraints in $r$ - $\alpha_{\text {iso }}$ and $\gamma_{\text {iso }}-\alpha_{\text {iso }}$ planes for the generally correlated case.

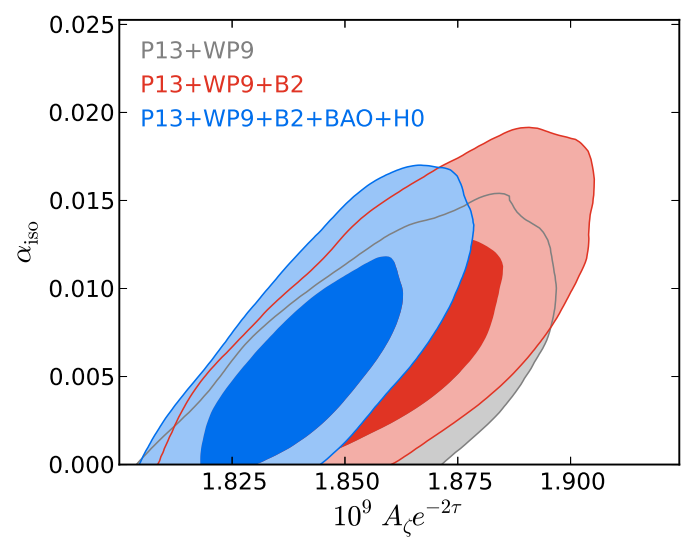

Figure 6: Degeneracy between $A_{\zeta} e^{-2 \tau}$ and $\alpha_{\text {iso }}$ in the anti-correlated isocurvature model.

Finally we discuss a case of uncorrelated isocurvature perturbations $\left(\gamma_{\text {iso }}=0\right)$ with $n_{\text {iso }} \neq n_{s}$, which we refer to as the varying $n_{\text {iso }}$ case. Here $n_{\text {iso }}$ can vary freely in the range $[0,4]$. Fig. [7 shows the 2D constraints in the $r$ - $\alpha_{\text {iso }}$ and the $n_{\text {iso }}-\alpha_{\text {iso }}$ planes. While a large 
fraction of isocurvature perturbations $\alpha_{\text {iso }} \simeq 0.4\left(\right.$ at $\left.k=0.05 \mathrm{Mpc}^{-1}\right)$ \#5 are allowed when the isocurvature spectrum is blue-tilted, we found no particular preference for $\alpha_{\text {iso }} \neq 0$. On the other hand, we found no significant improvements in the fits to data from the purely adiabatic case.
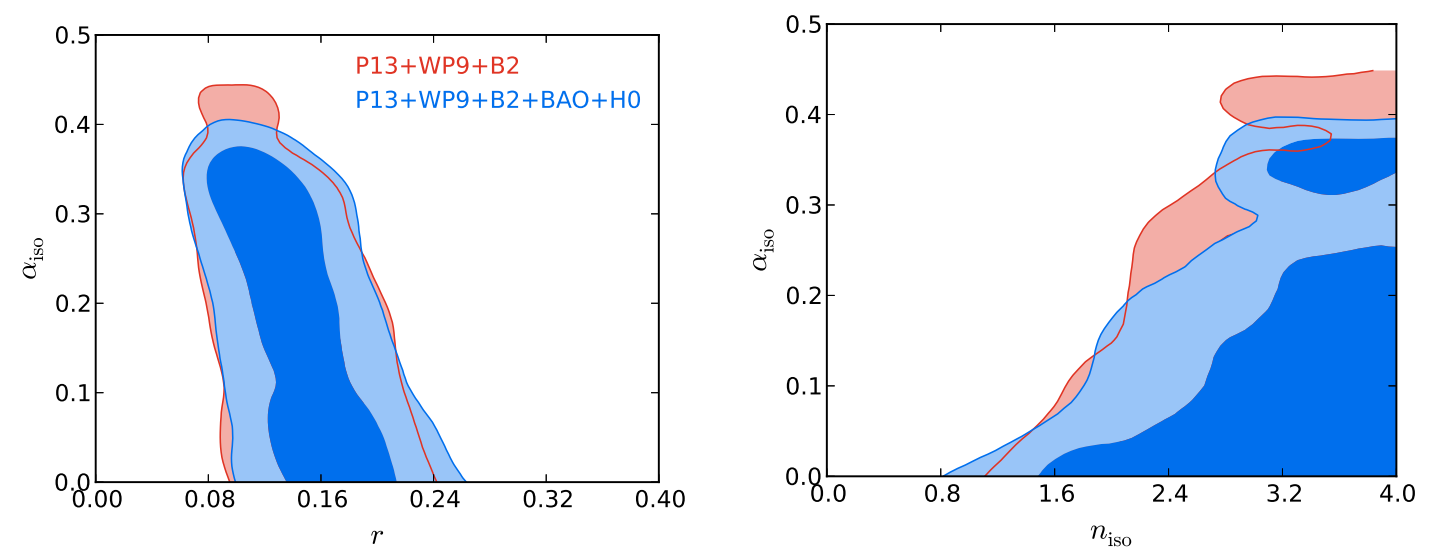

Figure 7: 2D constraints in the $r$ - $\alpha_{\text {iso }}$ and the $n_{\text {iso }}-\alpha_{\text {iso }}$ planes for the uncorrelated isocurvature case with varying $n_{\text {iso }}$.

\section{Conclusion}

In the conventional cosmology with adiabatic initial perturbations, there has been found a tension between the temperature data from Planck and the B-mode one from BICEP2. In this study, we have examined whether this tension can be mitigated by introducing isocurvature perturbations. We have found that, assuming the anti-correlated isocurvature perturbations, which can be realized in e.g. the curvaton scenario, $\chi^{2}$ is reduced by $\sim 6$ from the case of purely adiabatic perturbations and the constraints on $r$ from Planck alone and Planck combined with BICEP2 can be consistent at $68 \%$ C.L. However, while the level of discrepancy can be reduced, the current data is yet to show a clear evidence for nonvanishing isocurvature perturbations. We suggest that precise measurements of the CMB polarization in the future are promising to distinguish the anti-correlated isocurvature model from the adiabatic one.

\#5 We note that such large blue isocurvature perturbations can affect the structure formation at small scales and can be strongly constrained from reionization in spite of large theoretical uncertainties [41]. 


\section{Acknowledgments}

We would like to thank Jussi Valiviita and Matti Savelainen for valuable discussions and suggestions. The work is supported by Grant-in-Aid for Scientific Research 25400248 (MK), 23740195 (TT), 24-2775 (SY) and 21111006 (MK) from the Ministry of Education, Culture, Sports, Science and Technology in Japan, and also by World Premier International Research Center Initiative (WPI Initiative), MEXT, Japan. TS is supported by the Academy of Finland grant 1263714. We thank the CSC - IT Center for Science (Finland) for computational resources.

\section{References}

[1] P. A. R. Ade et al. [BICEP2 Collaboration], arXiv:1403.3985 [astro-ph.CO].

[2] P. A. R. Ade et al. [BICEP2 Collaboration], arXiv:1403.4302 [astro-ph.CO].

[3] P. A. R. Ade et al. [Planck Collaboration], arXiv:1303.5082 [astro-ph.CO].

[4] Y. -Z. Ma and Y. Wang, arXiv:1403.4585 [astro-ph.CO].

[5] M. Czerny, T. Kobayashi and F. Takahashi, arXiv:1403.4589 [astro-ph.CO].

[6] C. R. Contaldi, M. Peloso and L. Sorbo, arXiv:1403.4596 [astro-ph.CO].

[7] V. Miranda, W. Hu and P. Adshead, arXiv:1403.5231 [astro-ph.CO].

[8] K. N. Abazajian, G. Aslanyan, R. Easther and L. C. Price, arXiv:1403.5922 [astroph.CO].

[9] D. K. Hazra, A. Shafieloo, G. F. Smoot and A. A. Starobinsky, arXiv:1403.7786 [astro-ph.CO].

[10] E. Giusarma, E. Di Valentino, M. Lattanzi, A. Melchiorri and O. Mena, arXiv:1403.4852 [astro-ph.CO].

[11] J. -F. Zhang, Y. -H. Li and X. Zhang, arXiv:1403.7028 [astro-ph.CO].

[12] M. Gerbino, A. Marchini, L. Pagano, L. Salvati, E. Di Valentino and A. Melchiorri, arXiv:1403.5732 [astro-ph.CO].

[13] A. Ashoorioon, K. Dimopoulos, M. M. Sheikh-Jabbari and G. Shiu, arXiv:1403.6099 [hep-th].

[14] F. Wu, Y. Li, Y. Lu and X. Chen, arXiv:1403.6462 [astro-ph.CO].

[15] M. Kawasaki and S. Yokoyama, arXiv:1403.5823 [astro-ph.CO]. 
[16] M. Bastero-Gil, A. Berera, R. O. Ramos and J. G. Rosa, arXiv:1404.4976 [astroph.CO].

[17] K. Enqvist and M. S. Sloth, Nucl. Phys. B 626, 395 (2002) arXiv:hep-ph/0109214.

[18] D. H. Lyth and D. Wands, Phys. Lett. B 524, 5 (2002) arXiv:hep-ph/0110002.

[19] T. Moroi and T. Takahashi, Phys. Lett. B 522, 215 (2001) [Erratum-ibid. B 539, 303 (2002)] arXiv:hep-ph/0110096.

[20] T. Moroi and T. Takahashi, Phys. Rev. D 66, 063501 (2002) hep-ph/0206026.

[21] D. H. Lyth and D. Wands, Phys. Rev. D 68, 103516 (2003) astro-ph/0306500.

[22] M. Beltran, Phys. Rev. D 78, 023530 (2008) arXiv:0804.1097 [astro-ph]].

[23] T. Moroi and T. Takahashi, Phys. Lett. B 671, 339 (2009) arXiv:0810.0189 [hep$\mathrm{ph}]$.

[24] T. Takahashi, M. Yamaguchi and S. Yokoyama, Phys. Rev. D 80, 063524 (2009) arXiv:0907.3052 [astro-ph.CO]].

[25] M. Kawasaki and T. Sekiguchi, Prog. Theor. Phys. 120, 995 (2008) arXiv:0705.2853 [astro-ph]].

[26] J. Valiviita, M. Savelainen, M. Talvitie, H. Kurki-Suonio and S. Rusak, Astrophys. J. 753, 151 (2012) arXiv:1202.2852 [astro-ph.CO]].

[27] M. Savelainen, J. Valiviita, P. Walia, S. Rusak and H. Kurki-Suonio, Phys. Rev. D 88, 063010 (2013) arXiv:1307.4398 [astro-ph.CO]].

[28] S. Kasuya and M. Kawasaki, Phys. Rev. D 80, 023516 (2009) arXiv:0904.3800 [astro-ph.CO]].

[29] A. R. Liddle and D. H. Lyth, Phys. Rept. 231, 1 (1993) astro-ph/9303019.

[30] P. A. R. Ade et al. [Planck Collaboration], arXiv:1303.5076 [astro-ph.CO].

[31] A. Lewis and S. Bridle, Phys. Rev. D 66, 103511 (2002) astro-ph/0205436.

[32] A. Lewis, Phys. Rev. D 87, no. 10, 103529 (2013) arXiv:1304.4473 [astro-ph.CO]].

[33] P. A. R. Ade et al. [ Planck Collaboration], arXiv:1303.5072 [astro-ph.CO].

[34] P. A. R. Ade et al. [Planck Collaboration], arXiv:1303.5075 [astro-ph.CO].

[35] C. L. Bennett et al. [WMAP Collaboration], Astrophys. J. Suppl. 208, 20 (2013) arXiv:1212.5225 [astro-ph.CO]]. 
[36] G. Hinshaw et al. [WMAP Collaboration], Astrophys. J. Suppl. 208, 19 (2013) arXiv:1212.5226 [astro-ph.CO]].

[37] F. Beutler, C. Blake, M. Colless, D. H. Jones, L. Staveley-Smith, L. Campbell, Q. Parker and W. Saunders et al., Mon. Not. Roy. Astron. Soc. 416 (2011) 3017 arXiv:1106.3366 [astro-ph.CO]].

[38] L. Anderson, E. Aubourg, S. Bailey, D. Bizyaev, M. Blanton, A. S. Bolton, J. Brinkmann and J. R. Brownstein et al., Mon. Not. Roy. Astron. Soc. 427, no. 4, 3435 (2013) [arXiv:1203.6594 [astro-ph.CO]].

[39] N. Padmanabhan, X. Xu, D. J. Eisenstein, R. Scalzo, A. J. Cuesta, K. T. Mehta and E. Kazin, Mon. Not. Roy. Astron. Soc. 427, no. 3, 2132 (2012) arXiv:1202.0090 [astro-ph.CO]].

[40] A. G. Riess, L. Macri, S. Casertano, H. Lampeitl, H. C. Ferguson, A. V. Filippenko, S. W. Jha and W. Li et al., Astrophys. J. 730, 119 (2011) [Erratum-ibid. 732, 129 (2011)] arXiv:1103.2976 [astro-ph.CO]].

[41] T. Sekiguchi, H. Tashiro, J. Silk and N. Sugiyama, JCAP 1403, 001 (2014) arXiv:1311.3294 [astro-ph.CO]].

[42] J. Tauber et al. [Planck Collaboration], astro-ph/0604069.

[43] T. Matsumura, Y. Akiba, J. Borrill, Y. Chinone, M. Dobbs, H. Fuke, A. Ghribi and M. Hasegawa et al., arXiv:1311.2847 [astro-ph.IM].

[44] A. Kogut, D. J. Fixsen, D. T. Chuss, J. Dotson, E. Dwek, M. Halpern, G. F. Hinshaw and S. M. Meyer et al., JCAP 1107, 025 (2011) arXiv:1105.2044 [astro-ph.CO]].

[45] P. Andre et al. [PRISM Collaboration], arXiv:1306.2259 [astro-ph.CO]. 